\title{
Study of Rare-Earth Ions Implanted into Copper, Aluminum, Silver, and Rhodium*
}

\author{
D. W. Gebbie ${ }^{\dagger}$ C. Scherer, $\$$ D. L. Huber, G. M. Heestand,$\S$ and R. R. Borchers \\ University of Wisconsin, Madison, Wisconsin 53706
}

(Received 15 August 1972)

\begin{abstract}
Various even-even isotopes of $\mathrm{Er}, \mathrm{Dy}$, and $\mathrm{Yb}$ have been Coulomb excited to their first $2^{+}$states and implanted into metallic foils of $\mathrm{Cu}, \mathrm{Al}, \mathrm{Ag}$, and $\mathrm{Rh}$. The attenuation of the subsequent $\gamma$-ray angular distribution was measured as a function of temperature and interpreted in terms of a predominant time-dependent magnetic interaction with small admixtures of static and time-dependent electric interactions. Correlation times have been extracted and quantitively compared to the electronic relaxation times $\left(T_{2}\right)$ obtained from electron-paragmagnetic-resonance measurements of the same impurity-host systems. Comparisons are favorable, provided crystalline-field effects are taken into account.
\end{abstract}

\section{INTRODUCTION}

\section{A. General}

It has been known ${ }^{1-3}$ for several years that the angular correlation of the $\gamma$ rays from the first $2^{*}$ states (see Table I) of rare-earth nuclei implanted in copper appear strongly attenuated when studied with the ion-implantation perturbed-angular-correlation technique (IMPAC). Most of the existing data, with the exception of $\mathrm{Gd}$ and $\mathrm{Yb}$, can be explained by the existence of temporal fluctuations in the hyperfine field at the site of the rare-earth nucleus. Fluctuation times are known to be on the order of $10^{-12} \mathrm{sec}$ at room temperature. Static interactions are also present but in most cases are dominated by the time-dependentinteractions. Since most of the hyperfine interaction in rare-earth ions comes from the $4 f$ shell, it must be concluded that fluctuations in the hyperfine field arise from fluctuations in the angular momentum of the $4 f$ shell.

Reported here is the extension of the measurements to the cases of rare-earth ions implanted into $\mathrm{Al}, \mathrm{Ag}$, and $\mathrm{Rh}$. Attenuation coefficients were measured as a function of temperature between 77 and $450 \mathrm{~K}$. The time-dependent interaction is assumed to be explained by the simple Abragam and Pound ${ }^{4}$ theory as modified by Scherer ${ }^{5}$ to take into account admixtures of static quadrupole interactions. Correlation times are then extracted from the modified Abragam and Pound theory.

Temperature measurements from this paper and from Ref. 1 indicate that the reciprocal of the correlation time increases approximately linearly with temperature for $T>77 \mathrm{~K}$. This linear relation can be explained in terms of conduction electron exchange scattering from the $4 f$ shell. A similar effect has been observed in the electron-paramagnetic-resonance (EPR) linewidths ${ }^{6,7}$ for rare-earth ions in simple metals.

In this paper a quantitative effort is made to tie together the EPR linewidth data and the data on IMPAC correlation times. Reasonable agreement is obtained provided one includes the effects of the crystalline field.

\section{B. Experimental}

The IMPAC technique has been well documented ${ }^{8-10}$ for $g$-factor and hyperfine-field measurements in ferromagnetic materials. In these experiments the technique is nearly the same but a slight difference merits a brief discussion.

Figure 1 depicts the scattering geometry used in IMPAC. A 25-MeV oxygen beam produced by the University of Wisconsin tandem accelerator is used to Coulomb excite and implant rare-earth nuclei into a suitable host metallic polycrystalline foil. Implantation depths are on the order of $6000 \AA$ while stopping times are close to 1 psec. ${ }^{11}$ Final location is uncertain but existing information $^{12}$ for $\mathrm{Dy}$ in $\mathrm{Cu}$ and $\mathrm{Yb}$ in $\mathrm{Fe}$ indicates that $50 \%$ or more of the rare-earth ions go into substitutional sites. Although the impurity concentration is negligible $\left(<10^{-5} \%\right)$, radiation damage near the impurity site can be quite extensive.

The decay $\gamma$ rays from the Coulomb excited nuclei are detected by four $1 \frac{3}{4} \times 1 \frac{1}{2}$-in. NaI counters. The $\gamma$ rays are detected in coincidence with backscattered oxygen ions using a standard ring-counter geometry. Time resolution for the $80-\mathrm{keV} \gamma$ rays was close to $3 \mathrm{nsec}$. The coincidence requirement insures a highly anisotropic angular distribution and a deep implantation.

In general, the angular correlation between the decay $\gamma$ rays and the Coulomb-exciting oxygen ions can be written as an expansion in even Legendre polynomials:

$$
W(\theta, t)=1+A_{2} G_{2}(t) P_{2}(\cos \theta)+A_{4} G_{4}(t) P_{4}(\cos \theta) .
$$

The angle $\theta$ is measured with respect to the beam axis while $t=0$ is taken as the time the excited state is created. The parameters $A_{2}$ and $A_{4}$ are the angular correlation 'coefficients (including geometrical corrections) and are well known, while $G_{2}(t)$ and 
TABLE I. Relevant nuclear properties of the implanted nuclei.

\begin{tabular}{lcccc}
\hline \hline & $\begin{array}{c}\text { Energy of } \\
\text { first } 2^{+} \text {state } \\
\text { (keV) }\end{array}$ & $\begin{array}{c}\text { Mean } \\
\text { lifetime } \\
\text { (nsec) }\end{array}$ & $\begin{array}{l}\text { Magnetic } \\
\text { moment }^{\mathrm{b}} \\
\text { (nuclear } \\
\text { magnetons) }\end{array}$ & $\begin{array}{c}\text { Quadrupole } \\
\text { moment } \\
\text { (barns) }\end{array}$ \\
\hline${ }^{170} \mathrm{Er}$ & 79.3 & 2.73 & 0.66 & $1.36^{\mathrm{b}}$ \\
${ }^{162} \mathrm{Dy}$ & 80.7 & 3.18 & 0.71 & $2.04^{\mathrm{c}}$ \\
${ }^{174} \mathrm{Yb}$ & 76.5 & 2.58 & 0.64 & $2.23^{\mathrm{c}}$ \\
\hline \hline
\end{tabular}

${ }^{a}$ Weighted average of all known direct measurements.

${ }^{b}$ Weighted average of all measurements reported in Ref. 19

${ }^{c}$ Calculated from the $B(E 2)$ values given in Ref. 15 .

$G_{4}(t)$ are the attenuation coefficients. In this paper $G_{2}(t)$ and $G_{4}(t)$ were measured both as a function of time (time-differential mode) and averaged over the nuclear lifetime (time-integral mode). Mathematically this is described as

$$
\bar{G}_{k}^{\infty}=\frac{1}{\tau_{N}} \int_{0}^{\infty} G_{k}(t) e^{-t / \tau_{N}} d t, \quad k=2,4 .
$$

Most measurements were done in the integral mode because it represents a considerable saving in experimental time over the differential mode. The time-differential data was used only as a check on the exponential decay of the angular-correlation coefficients.

Integral measurements of $G_{2}$ and $G_{4}$ were carried out as a function of temperature for ${ }^{170} \mathrm{Er}$, ${ }^{162} \mathrm{Dy}$, and ${ }^{174} \mathrm{Yb}$ implanted into $\mathrm{Cu}, \mathrm{Ag}, \mathrm{Al}$, and $\mathrm{Rh}$ foils. Temperatures down to $77 \mathrm{~K}$ were obtained using a cold finger attached to a cold trap. By placing a heater on the cold finger close to the target the temperature of the target could easily be raised to $200 \mathrm{~K}$ when the cold trap was filled with

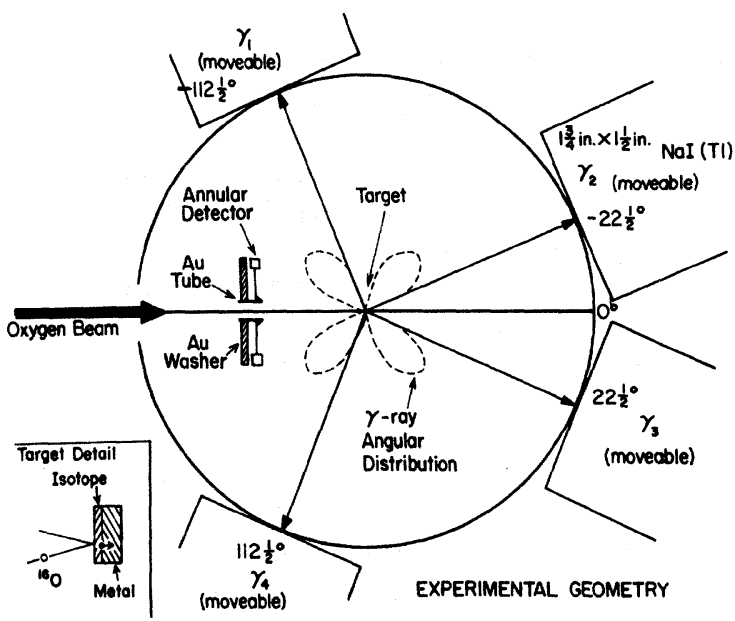

FIG. 1. Schematic of the scattering geometry, top view. The dotted cloverleaf represents an unattenuated $\gamma$-ray angular distribution from a $2^{+} \rightarrow 0^{+}$transition when the $2^{+}$state is Coulomb excited by only backscattered oxgyen ions. liquid nitrogen. Temperatures as high as $450 \mathrm{~K}$ were obtained by using the heater with the cold trap empty. Temperatures were measured with an iron-constantan thermocouple placed about $1 \mathrm{~cm}$ above the beam spot. Beam-heating effects have been measured ${ }^{13}$ and are on the order of a few degrees centigrade.

\section{EXPERIMENTAL RESULTS}

\section{A. General}

A summary of the integral measurements $\bar{G}_{2}^{\infty}$ and $\bar{G}_{4}^{\infty}$ is shown in Fig. 2. This represents about two thirds of our measurements, the rest being omitted because they nearly overlap the existing points on the figure. Variations in temperature produce the range in the $\bar{G}_{2}^{\infty}$ and $\bar{G}_{4}^{\infty}$ values.

The solid curve in Fig. 2 represents the theory of Abragam and Pound ${ }^{4}$ for a pure time-dependent magnetic interaction. Abragam and Pound predict that for nuclei under the influence of a fluctuating magnetic hyperfine interaction $G_{2}(t)$ and $G_{4}(t)$ will be exponentially varying with

$$
\begin{aligned}
& G_{k}(t)=e^{-\lambda_{k} t}, \\
& \lambda_{k}=\frac{1}{3} k(k+1) J(J+1)(A / \hbar)^{2} \tau_{c}, \\
& \bar{G}_{k}^{\infty}=1 /\left(1+\lambda_{k} \tau_{N}\right),
\end{aligned}
$$

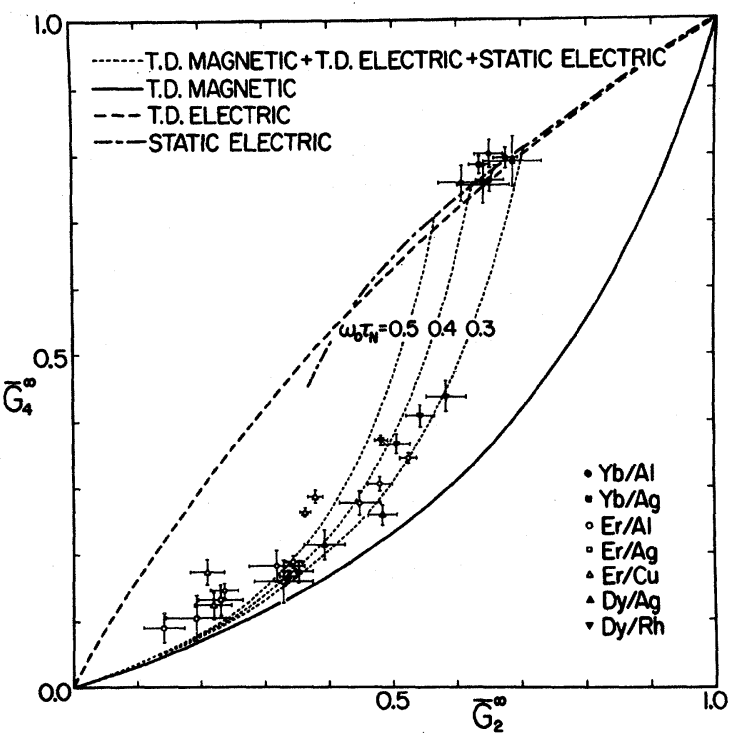

FIG. 2. Summary of the data taken in the integral mode. The spread in experimental points for one impurity-host system was obtained by varying the target temperature. The solid and dashed lines represent timedependent magnetic and electric interactions, respectively, using the Abragam and Pound model. The dot-dashed line represents a static randomly oriented electric quadrupole interaction. The dotted line is a combination of all three for different values of $\omega_{0} \tau_{N}$, the static quadrupole frequency times the nuclear lifetime. 
where the hyperfine interaction is assumed to be of the form $H=A \overrightarrow{\mathrm{I}} \cdot \overrightarrow{\mathrm{J}}$ in which $\overrightarrow{\mathrm{I}}$ is the nuclear spin and $\hbar \vec{J}$ is the electronic angular momentum. Time $t=0$ is the creation time of the excited state, $\tau_{N}$ is the state's lifetime, and $\tau_{c}$, the correlation time, is a measure of the time between fluctuations in the hyperfine interaction. For the theory to be valid. it is necessary to assume that $\tau_{c} \ll \tau_{N}$ and $(A / \hbar)$ $\tau_{c} \ll 1$. As will be pointed out later both conditions are met. The solid line is a plot of $\bar{G}_{4}^{\infty}$ versus $\bar{G}_{2}^{\infty}$, with $\lambda_{4}=\frac{10}{3} \lambda_{2}$ as the free parameter.

The dashed line represents the case of a pure time-dependent electric interaction. For this case Abragam and Pound also predict an exponential decay in the $G_{k}(t)$ with

$$
\lambda_{k}=\frac{3}{80} \tau_{c}\left(\frac{e Q V_{g z}}{\hbar}\right)^{2} \frac{k(k+1)[4 I(I+1)-k(k+1)-1]}{I^{2}(2 I-1)^{2}} \text {. }
$$

Here $Q$ is the excited-state quadrupole moment, $V_{z z}$ is the fluctuating electric field gradient, and $I$ is the spin of the excited state.

The main signature of the time-dependent interaction, at least when $G_{2}$ and $G_{4}$ are measured in the integral mode, is the absence of a hard core, i. e., both $\bar{G}_{2}^{\infty}$ and $\bar{G}_{4}^{\infty}$ can go to zero. Both randomly oriented pure-static-electric and purestatic-magnetic interactions give rise to finite lower limits on $\bar{G}_{2}^{\infty}$ and $\bar{G}_{4}^{\infty}$.

The dot-dash line in Fig. 2 represents the randomly oriented static quadrupole interaction given by

$$
\begin{aligned}
& G_{k}(t)=\sum_{n} S_{k n} \cos n \omega_{0} t, \quad \bar{G}_{k}^{\infty}=\sum_{n} \frac{S_{k n}}{1+\left(n \omega_{0} \tau_{N}\right)^{2}} \\
& \omega_{0}= \begin{cases}3 \omega_{Q} I \text { even } & \omega_{Q}=-\frac{e Q V_{z z}}{4 I(2 I-1) \hbar}, \\
6 \omega_{Q} I \text { odd } & \end{cases}
\end{aligned}
$$

where the $S_{k n}$ 's are tabulated in Siegbahn. ${ }^{14}$ The dot-dashed line terminates at $\left(\bar{G}_{2}^{\infty}=0.3714, \bar{G}_{4}^{\infty}\right.$ $=0.4063$ ), the hard-core value for the $I=2$ case of the static electric interaction.

The data points for ${ }^{170} \mathrm{Er}$ and ${ }^{162} \mathrm{Dy}$ lie closest to the curve representing the time-dependent magnetic interaction. However, as can be seen, agreement between the data and a pure time-dependent magnetic interaction is poor. Much better agreement can be obtained by allowing for static electric and time-dependent electric interaction in addition to the time-dependent magnetic interaction.

A clue to the magnitude of the static electric interaction can be obtained from the data for ${ }^{174} \mathrm{Yb}$ in $\mathrm{Al}$ and $\mathrm{Ag}$. From Fig. 2 it appears that ${ }^{174} \mathrm{Yb}$ in $\mathrm{Al}$ and $\mathrm{Ag}$ can be explained by a pure static quadrupole interaction. From this it is inferred that no time-dependent interaction is present, so that the $\mathrm{Yb}$ ion has a filled $4 f$ shell and is in a $2+$ ionic state. The fact $\bar{G}_{2}^{\infty}$ and $\bar{G}_{4}^{\infty}$ are not unity means that the ion is not in a site of cubic symmetry.
This can arise either from the ions going into interstitial sites or from radiation damage, for $\mathrm{Al}$ and $\mathrm{Ag}$ have cubic structure. Using our measurements of $\bar{G}_{2}^{\infty}$ and $\bar{G}_{4}^{\infty}$ for ${ }^{174} \mathrm{Yb}$ in $\mathrm{Al}$ and $\mathrm{Ag}$ and the known quadrupole moment ${ }^{15}$ of ${ }^{174} \mathrm{Yb}$ we obtain an electric field gradient of $V_{z z}=3.5 \times 10^{17} \mathrm{~V} / \mathrm{cm}^{2}$. This field gradient is an average over all sites and is probably nonaxial.

It is very likely that the implanted $\mathrm{Er}$ and Dy nuclei see an average field gradient close to 3.5 $\times 10^{17} \mathrm{~V} / \mathrm{cm}^{2}$ along with a large time-dependent interaction.

Scherer ${ }^{5}$ has derived a functional form for $G_{k}(t)$ in the presence of both time-dependent magnetic and static electric interactions. To within a few percent his $G_{k}(t)$ reduce to $G_{k}(t)=G_{k}^{\text {(SE) }}(t) G_{k}^{\text {(TDM) }}(t)$, i. e., a simple product of the attenuation coefficients due to pure static electric (SE) and pure time-dependent magnetic (TDM) interactions.

The dotted lines in Fig. 2 represent the modified $G_{k}(t)$ for different values of $\omega_{0} \tau_{N}$. A small amount of time-dependent electric interaction has been added since a fluctuating $4 f$ shell also produces a fluctuating electric field gradient. The strength of the added time-dependent electric interaction was calculated using the $4 f$ field gradients of Günther ${ }^{16}$ and the known quadrupole moments. ${ }^{15}$

The curves generated assuming the presence of time-dependent electric and magnetic and static electric interactions fit the data much better than any single interaction. However, deviations still occur, especially for low values of $\bar{G}_{2}^{\infty}$ and $\bar{G}_{4}^{\infty}$. A good explanation for this is not known but one possibility is that there is more time-dependent electric and less static electric interaction than we have estimated.

\section{B. Temperature Measurements}

Measurements of $\bar{G}_{2}^{\infty}$ and $\bar{G}_{4}^{\infty}$ have been carried out for ${ }^{170} \mathrm{Er}$ in $\mathrm{Cu}, \mathrm{Al}$, and $\mathrm{Ag}$ and ${ }^{162} \mathrm{Dy}$ in $\mathrm{Ag}$ and $\mathrm{Rh}$. Values of $\lambda_{4}$ were extracted from the $\bar{G}_{4}^{\infty}$ data using the modified functional form of $\bar{G}_{4}^{\infty}$ to take into account electric quadrupole interactions. For the static quadrupole interaction, a $V_{z z}$ of $3.5 \times 10^{17}$ $\mathrm{V} / \mathrm{cm}^{2}$ was assumed, whereas for the time-dependent electric interaction the Günthe ${ }^{16}$ values of $V_{z z}$ were used. In all cases the effect of electric quadrupole interaction upon $\lambda_{4}$ values was considerably less than the effect upon $\lambda_{2}$ values.

In Fig. 3 is shown a plot of $1 / \lambda_{4}$ versus temperature. Since $\lambda_{4}$ is proportional to the correlation time $\tau_{c}$, it is apparent from Fig. 3 that $\tau_{c}^{-1}$ varies approximately linearly with temperature for $T>77 \mathrm{~K}$.

Values of $\tau_{c}$ were obtained directly from $\lambda_{4}$ using Eq. (3). Values for $A$ were obtained from $\mathrm{EPR}^{17,18}$ data by multiplying by the ratio of the nuclear $g$ factors. That is, we took 


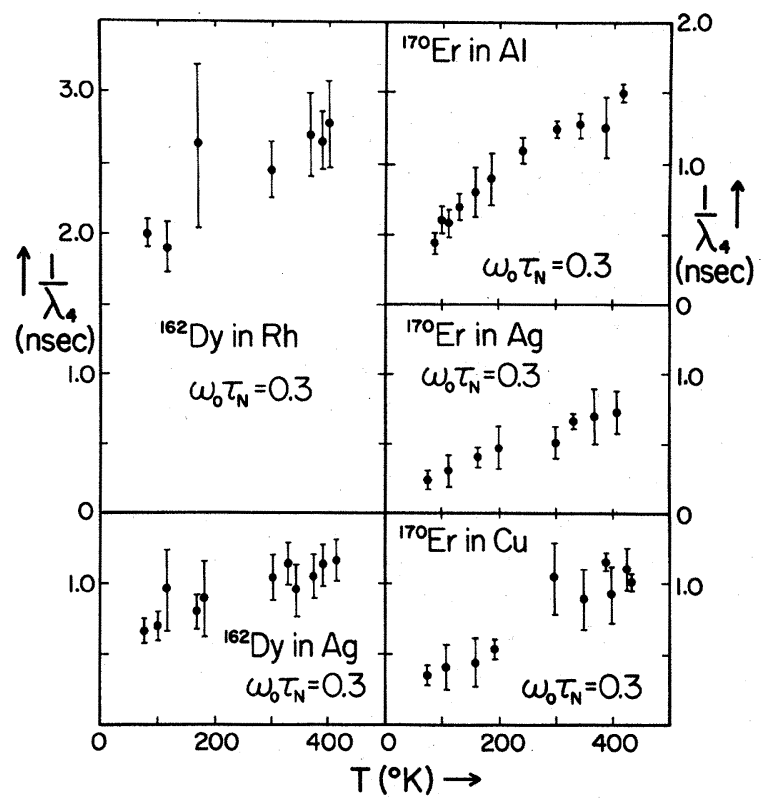

FIG. 3. Values of $1 / \lambda_{4}$ plotted vs temperature. A static electric quadrupole interaction of $\omega_{0} \tau_{N}=0.3$ was used in determining $\lambda_{4}$ from $\bar{G}_{4}^{\infty}$.

$$
\begin{aligned}
A\left({ }^{170} \mathrm{Er}\right) & =\frac{g_{n}\left({ }^{170} \mathrm{Er}\right) A\left({ }^{167} \mathrm{Er}\right)}{g_{n}\left({ }^{167} \mathrm{Er}\right)} \\
& =(1.73 \pm 0.06) \times 10^{-18} \mathrm{ergs}, \\
A\left({ }^{162} \mathrm{Dy}\right) & =\frac{g_{n}\left({ }^{162} \mathrm{Dy}\right) A\left({ }^{163} \mathrm{Dy}\right)}{g_{n}\left({ }^{163} \mathrm{Dy}\right)} \\
& =(1.31 \pm 0.05) \times 10^{-18} \mathrm{ergs},
\end{aligned}
$$

using values of $g_{n}$ which have been reported previously. ${ }^{19}$

Figures 4 and 5 show the experimental values of $\tau_{c}$ multiplied by the temperature $T$ plotted against $T$ for ${ }^{170} \mathrm{Er}$ in $\mathrm{Ag}$ and ${ }^{162} \mathrm{Dy}$ in $\mathrm{Ag}$. The solid lines in Figs. 4 and 5 are the results of theoretical calculations based upon existing $\mathrm{EPR}^{6,7}$ linewidth data and are explained in detail in Part III.

\section{Ytterbium}

As was pointed out in Sec. II A the $\bar{G}_{2}^{\infty}$ and $\bar{G}_{4}^{\infty}$ data for ${ }^{174} \mathrm{Yb}$ in $\mathrm{Al}$ and $\mathrm{Ag}$ are characteristic of a pure static quadrupole interaction. Temperature measurements for both ${ }^{174} \mathrm{Yb}$ in $\mathrm{Al}$ and $\mathrm{Ag}$ show that the interaction frqeunecy $\omega_{0}$ increases by about $10 \%$ in going from 450 to $77 \mathrm{~K}$. This seems to be consistent with the idea that $\mathrm{Yb}$ in $\mathrm{Al}$ and $\mathrm{Ag}$ is in a $2^{+}$ionic state and hence exhibits no time-dependent interaction.

In Fig. 6(a) is shown a plot of $G_{4}(t)$ vs $G_{2}(t)$ for ${ }^{174} \mathrm{Yb}$ in $\mathrm{Al}$. This plot was obtained from data taken in the time-differential mode and represents a time span of around $10 \mathrm{nsec}$. As can be seen the data are completely consistent with a pure static quadrupole interaction.

In Fig. 6(b) is shown the same plot as in Fig. 6(a) except for ${ }^{174} \mathrm{Yb}$ in $\mathrm{Cu} .{ }^{2}$ There is a clear deviation from the pure electric quadrupole interaction. Temperature measurements for ${ }^{174} \mathrm{Yb}$ in $\mathrm{Cu}$ indicate a much larger variation in the $\bar{G}_{k}^{\infty}$ with temperature. From this it is concluded that at least some of the ${ }^{174} \mathrm{Yb}$ nuclei are under the influence of a fluctuating hyperfine interaction. Consequently, $\mathrm{Yb}$ is present in a $3^{+}$ionic state when implanted into $\mathrm{Cu}$.

It is interesting to note that $\mathrm{Yb}$ in $\mathrm{Au}$ has been observed by $\mathrm{TaO}^{7}$ to be paramagnetic and hence in a $3^{+}$ionic state, whereas $\mathrm{Yb}$ in $\mathrm{Yb}$ metal is known to be in a $2^{+}$ionic state. Chuhran ${ }^{20}$ has shown using the perturbed angular correlation technique that ${ }^{172} \mathrm{Yb}$ in $\mathrm{Tm}$ metal is also in a $2^{+}$ionic state.

\section{CALCULATION OF $\tau_{c}$}

As discussed in Sec. II A, the coefficients $\lambda_{k}$ associated with the fluctuating hyperfine field can

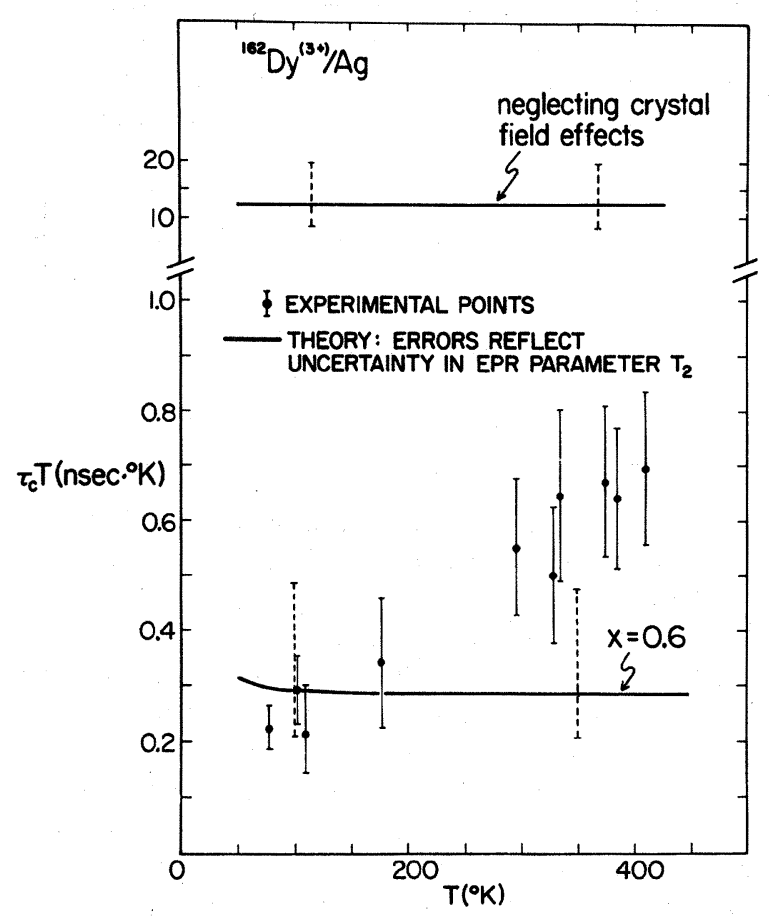

FIG. 4. Experimental values of $\tau_{c} T$ plotted vs temperature for ${ }^{162} \mathrm{Dy}^{(3+)}$ in $\mathrm{Ag}$. The solid lines represent theoretical values based upon the EPR relaxation times $T_{2}$ of Davidov (Ref. 6). The top line assumes no crystalline-field effects while the bottom line assumes that crystalline-field effects are present. The quantity " $x$ " compares the sixth-order and fourth-order cubic field terms and is defined in Ref. 22. A value of 0.6 for $x$ was determined from the work of Williams and Hirst (Ref. 21). The dotted error bar represents the error in the solid line and reflects the error in $T_{2}$. 
be written

$$
\lambda_{k}=\frac{1}{3}(A / \hbar)^{2} J(J+1) k(k+1) \tau_{c}
$$

for a scalar hyperfine interaction $A \overrightarrow{\mathrm{I}} \cdot \overrightarrow{\mathrm{J}}$. In the cases of cubic or spherical symmetry the parameter $\tau_{c}$, loosely identified as a correlation time, is expressed formally by the integral ${ }^{4}$

$$
\tau_{c}=\frac{1}{2}\left[\frac{1}{3} J(J+1)\right]^{-1} \int_{-\infty}^{\infty}\left\langle J_{z} J_{z}(t)\right\rangle d t
$$

where the brackets \langle\rangle denote a thermal average and $J_{z}(t)$ is given by

$$
J_{z}(t)=e^{i H t / \hbar} J_{z} e^{-i H t / \hbar},
$$

with $H$ being the electronic Hamiltonian.

The purpose of this section is to outline the analysis of $\tau_{c}$. It will be shown that the fluctuations in the electronic angular momentum almost certainly arise from the exchange scattering of the conduction electrons. A connection will be established between $\tau_{c}$ and the EPR linewidth associated with the ground manifold of the rare-earth ion. By exploiting this connection "theoretical" values are obtained for $\tau_{c}$ which compare favorably with experiment in the two cases studied in detail, ${ }^{170} \mathrm{Er}^{(3+)}$ and ${ }^{162} \mathrm{Dy}^{(3+)}$ in silver.

Before entering into the details of the analysis it should be pointed out that Eqs. (7) and (8), al-

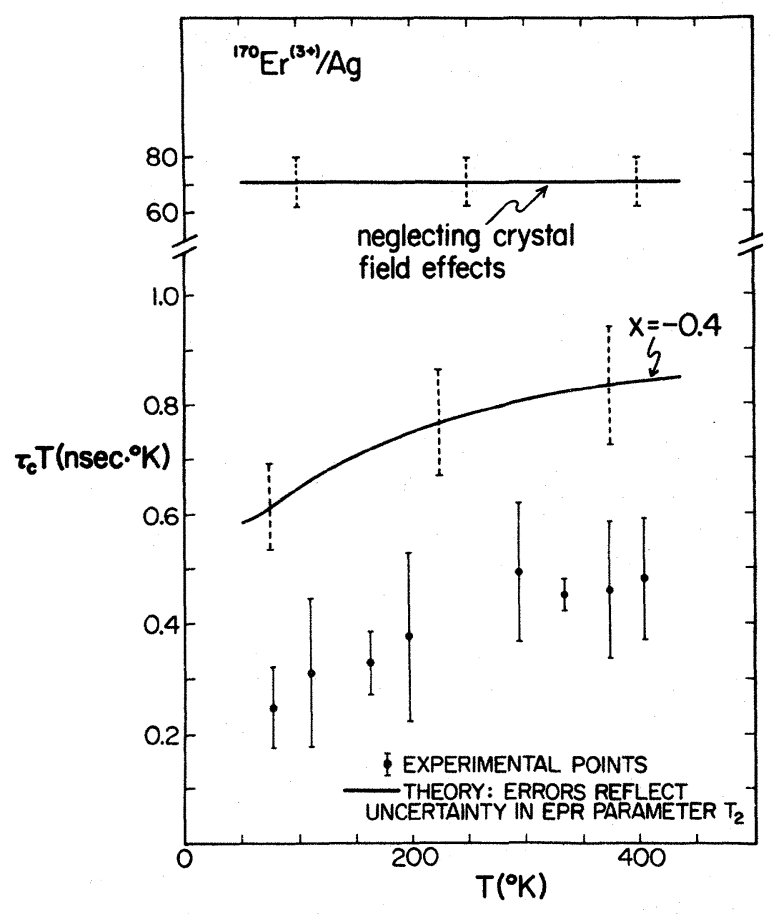

FIG. 5. Experimental values of $\tau_{c} T$ plotted vs temperature for ${ }^{170} \mathrm{Er}^{(3+)}$ in $\mathrm{Ag}$. The solid lines represent theoretical values based upon the EPR relaxation times $T_{2}$ of Davidov (Ref. 7)。The top line assumes no crystalline-field effects while the bottom line assumes that crystalline-field effects are present with $x=-0.4$.
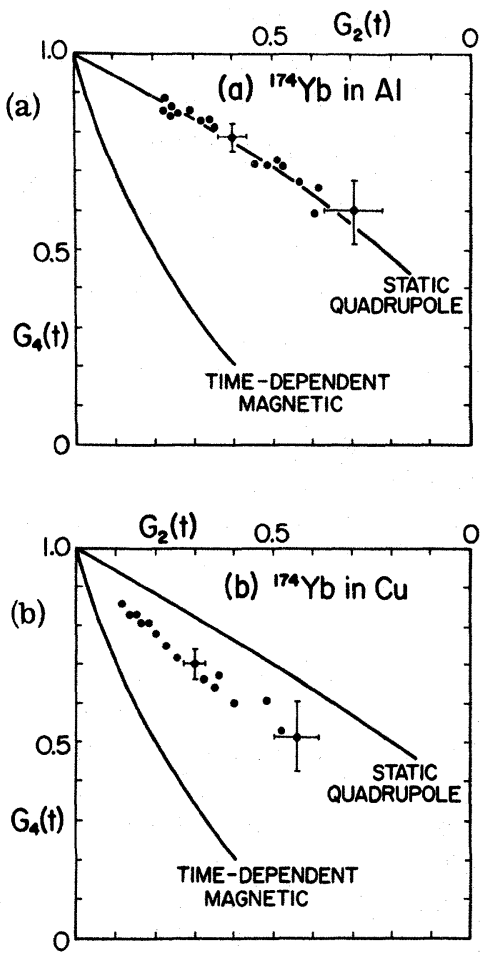

FIG. 6. (a) $G_{2}(t)$ vs $G_{4}(t)$ for ${ }^{174} \mathrm{Yb}$ in Al. The data were taken in the time differential mode and represent a time span of about 10 nsec. The top line corresponds to a static electric interaction while the bottom corresponds to a time-dependent magnetic interaction. (b) $G_{2}(t)$ vs $G_{4}(t)$ for ${ }^{174} \mathrm{Yb}$ in $\mathrm{Cu}$.

though appearing to be quite general, nevertheless have important limitations on their applicability. In (7), as was mentioned, it is assumed that $(A / \hbar) \tau_{c} \ll 1$; that is to say, the short-correlationtime limit applies. The second assumption is connected with the appearance of the thermal average in Eq. (8). For this to be appropriate it is necessary that the time it takes for the $4 f$ shell to come to thermal equilibrium with its environment is much shorter than the nuclear lifetime $\tau_{N}$. Since the mechanism responsible for $\tau_{c}$ also gives rise to thermal relaxation this second condition is equivalent to $\tau_{c} \ll \tau_{N}$. In the systems studied it was found that $(A / \hbar) \approx 10^{9} \mathrm{rad} \mathrm{sec}^{-1}, \tau_{N} \approx 3 \times 10^{-9} \mathrm{sec}$, while $\tau_{c} \lesssim 10^{-11} \mathrm{sec}$, so that both conditions are satisfied.

As noted, Eqs. (7) and (8) are, strictly speaking, appropriate only for cubic symmetry. When the symmetry is lower than cubic allowance must be made for a tensor coupling between $\overrightarrow{\mathrm{I}}$ and $\overrightarrow{\mathrm{J}}$ as well as for a variety of correlation times associated with the várious components of the angular momentum. It was mentioned previously that there is an electric field gradient at the rare-earth sites, which points to the absence of perfect cubic 
symmetry. The theoretical expressions for $\tau_{c}$ will be derived assuming the rare-earth ions are at cubic sites. However, the results are quantitatively insensitive to small deviations from cubic symmetry and appear to remain qualitatively correct for large deviations provided the strength of the coupling between the rare-earth ions and the conduction electrons is approximately the same as at the cubic sites.

The formal analysis of $\tau_{c}$ begins with the integral in Eq. (8). In evaluating this integral the influence of the hyperfine interaction on the dynamics of the electronic angular momentum can be neglected. This being the case the Hamiltonian $H$ that appears in Eq. (9) is of the form

$$
H=H_{\mathrm{CF}}+H_{\text {Int }} \text {, }
$$

where $H_{C F}$ is the interaction with the static crystal field and $H_{\text {Int }}$ is the part responsible for the dynamics. In insulators the orbit-lattice interaction causes the fluctuations in $\vec{J}$, which arise from the scattering of phonons by the paramagnetic ion. In metals the orbit-lattice mechanism is present but often is obscured by the exchange scattering of the conduction electrons. Since the correlation time associated with phonon scattering is a rapidly varying function of temperature (typically $T^{-7}$ or $e^{a / T}$ for $T>10 \mathrm{~K}$ ) whereas $\tau_{c}$ for electron scattering is approximately proportional to $T^{-1}$, it is often possible to decide which of the two mechanisms is the more important on the basis of the temperature dependence of $\tau_{c}$ alone. Such appears to be the case with our measurements which point to conduction electron scattering, a result substantiated by the analysis.

In the calculation of $\tau_{c}$ only the lowest spin-orbit multiplet of the rare-earth ion, assumed to be in the trivalent state, is considered. The crystal field splits the $2 J+1$ degenerate levels into crystal field multiplets labeled by $\Gamma, n(\Gamma)$, where $\Gamma$ denotes a particular representation of the cubic group and $n(\Gamma)$ labels the levels within that representation. The role of $H_{\text {Int }}$ is then to induce transitions between the various crystal field levels. To see how this comes about the correlation function $\left\langle J_{z} J_{z}(t)\right\rangle$ is expanded in terms of the states $|n(\Gamma) \Gamma\rangle$ :

$$
\begin{aligned}
\left\langle J_{z} J_{z}(t)\right\rangle=\sum_{\Gamma, \Gamma^{\prime}} \sum_{n(\Gamma), n\left(\Gamma^{\circ}\right)} P_{\Gamma}\left\langle\Gamma n(\Gamma)\left|J_{z}\right| n\left(\Gamma^{\prime}\right) \Gamma^{\prime}\right\rangle \\
\quad \times\left\langle\Gamma^{\prime} n\left(\Gamma^{\prime}\right)\left|J_{z}(t)\right| n(\Gamma) \Gamma\right\rangle, \quad \text { (11) }
\end{aligned}
$$

where $P_{\Gamma}$ is the probability that a level in the $\Gamma$ manifold is occupied. It is given by

$$
P_{\Gamma}=\frac{e^{-E} \Gamma^{/ k T}}{\sum_{\Gamma} \sum_{n(\Gamma)} e^{-E} \Gamma^{/ k T}}
$$

Qualitative insight into the calculation of $\tau_{c}$ can be gained by making the approximation

$$
\begin{aligned}
\left\langle\Gamma^{\prime} n\left(\Gamma^{\prime}\right)\left|J_{z}(t)\right| n(\Gamma) \Gamma\right\rangle & -\left\langle\Gamma^{\prime} n\left(\Gamma^{\prime}\right)\left|J_{z}\right| n(\Gamma) \Gamma\right\rangle \\
& \times e^{i\left(E_{\Gamma},-E\right.} \Gamma^{\prime t / \hbar} e^{-|t| / \bar{\tau}} .
\end{aligned}
$$

This approximation amounts to assuming that the scattering induces an exponential decay in the $z$ component of the electronic angular momentum. The rate of decay $\bar{\tau}$ is a typical "average" correlation time, and is on the order of $\tau_{c}$. With this form for the matrix element $\tau_{c}$ is given by

$$
\begin{aligned}
\tau_{c}= & {\left[\frac{1}{3} J(J+1)\right]^{-1} } \\
& \times \sum_{\Gamma, \Gamma^{\prime}} \sum_{n(\Gamma), n\left(\Gamma^{\circ}\right)} P_{\Gamma}\left|\left\langle\Gamma n(\Gamma)\left|J_{z}\right| n\left(\Gamma^{\prime}\right) \Gamma^{\prime}\right\rangle\right|^{2} \\
& \times \bar{\tau} /\left[1+\bar{\tau}^{2}\left(E_{\Gamma}-E_{\Gamma^{\prime}}\right)^{2} \hbar^{-2}\right]
\end{aligned}
$$

Apart from accidental degeneracies, which are almost always removed by the noncubic perturbations, the splittings between different crystal-field manifolds are large in comparis on with $\hbar / \bar{\tau}$ for realistic values of $\tau_{c}$. As a consequence only the terms with $\Gamma^{\prime}=\Gamma$ make important contributions to $\tau_{c}$. Thus $\tau_{c}$ becomes

$$
\begin{aligned}
\tau_{c} & \approx\left[\frac{1}{3} J(J+1)\right]^{-1} \\
& \times \sum_{\Gamma} \sum_{n(\Gamma), n^{\circ}(\Gamma)} P_{\Gamma}\left|\left\langle\Gamma n(\Gamma)\left|J_{z}\right| n^{\prime}(\Gamma) \Gamma\right\rangle\right|^{2} \tau_{\Gamma},
\end{aligned}
$$

where the results of the Appendix have been anticipated by allowing for a correlation time associated with each manifold. [In this equation $n(\Gamma)$ and $n^{\prime}(\Gamma)$ label various sublevels in the $\Gamma$ manifold.] Equation (15) is to be compared with the expression obtained when the crystal-field splittings are set equal to zero. In this limit Eq. (14) reduces to

$$
\begin{aligned}
\tau_{c}= & {\left[\frac{1}{3} J(J+1)\right]^{-1} \sum_{\Gamma, \Gamma^{\prime}} \sum_{n(\Gamma), n\left(\Gamma^{\prime}\right)}[\bar{\tau} /(2 J+1)] } \\
& \times\left.\left\langle\Gamma n(\Gamma)\left|J_{z}\right| n\left(\Gamma^{\prime}\right) \Gamma^{\prime}\right\rangle\right|^{2} \\
= & \bar{\tau} .
\end{aligned}
$$

The calculation of the parameters $\tau_{\Gamma}$ appearing in Eq. (15) is outlined in the Appendix. The expression that is obtained for $1 / \tau_{\Gamma}$, Eqs. (A8) and (A9), is seen to be of the form

$$
1 / \tau=T C_{e 1} f(\Gamma, T),
$$

where $C_{\mathrm{e} 1}$ is a temperature-independent multiplicative factor which is proportional to the square of the exchange integral connecting the rare-earth spin and the spin of the conduction electrons. The function $f(\Gamma, T)$ depends only on temperature and the crystal-field parameters but varies from manifold to manifold, in a way which is easily calculable

Rather than calculate $C_{e 1}$ directly it is preferable to obtain an empirical value by making use of the fact that $\tau_{\Gamma}$ is equal to $T_{2}$ the low-field EPR linewidth for the $\Gamma$ manifold. By fitting the ob- 
served linewidth to the functional form shown in Eq. (17) an "experimental" value for $C_{\text {el }}$ can be obtained which can then be used in the calculation of the correlation times for all of the manifolds.

Because of the availability of crystal-field data attention has been focused on Dy and Er in silver. Values of the crystal-field parameters for the cubic sites in silver have been published by Williams and Hirst. ${ }^{21}$ Also, measurements of the EPR linewidth of the $\Gamma_{7}$ multiplet have been reported for $\mathrm{Dy}^{(3+)}$ by Davidov et al. , ${ }^{6}$ and for $\operatorname{Er}^{(3+)}$ by Davidov et al. ${ }^{7}$ The calculation of $\tau_{c}$ was carried out using the crystal-field wave functions of Lea, Leask, and Wolf. ${ }^{22}$ The parameter $x$, defined in Lea, Leask, and Wolf, was determined from the Williams and Hirst data $(x \approx-0.4$ for $\mathrm{Er}$ and $x \approx 0.6$ for Dy). Values of $C_{e 1}$ were inferred by fitting the experimental values for the temperature dependent term in the linewidth to the theoretical expression for $\tau_{\Gamma 7}$ evaluated at $4 \mathrm{~K}$.

The results of the theoretical analysis using the EPR data are shown along with the angular correlation data in Figs. 4 and 5, where we have plotted $\tau_{c} T$ vs $T$. The theoretical curves were obtained from Eqs. (15), (A8), and (A9). Also shown are the values that were found when degenerate free-ion states were used in place of crystalfield states in the evaluation of $\tau_{c}, C_{e 1}$ remaining the same. In each case the uncertainties in $\tau_{c} T$ reflect the uncertainties in the experimental data for $T_{2}$ and hence $C_{e 1}$.

It is seen that for both ions the experimental and theoretical curves agree to within a factor of about 2. A difference of this magnitude is not unexpected considering the variation in crystal-field parameters and exchange integrals arising from the distribution in rare-earth sites. Somewhat surprising and not understood is the observed temperature variation of the experimental values of $\tau_{c} T$ in Dy. One of the more significant features of the results is the comparison between the "freeion" values for $\tau_{c} T$ and the values obtained with the crystal-field wave functions. With Dy the two values differ by more than a factor of 40 , where as in the case of Er the ratio is closer to one hundred. In both cases the size of the ratio is a dramatic indication of the importance of including crystal-field effects in the analysis of $\tau_{c}$.

\section{SUMMARY}

The results of this paper fall into two categories. First, experimental data on the decay of the angular correlation were reported for ${ }^{174} \mathrm{Yb},{ }^{170} \mathrm{Er}$, and ${ }^{162} \mathrm{Dy}$ implanted into $\mathrm{Al}, \mathrm{Ag}, \mathrm{Cu}$, and $\mathrm{Rh}$. The contributions to the decay arising from time-dependent-magnetic, time-dependent-electric, and static-electric interactions were established. Second, a detailed theoretical analysis of the corre- lation time of the time-dependent-magnetic interaction was carried out for $\mathrm{Er}$ and $\mathrm{Dy}$ in $\mathrm{Ag}$. The conclusion of this analysis was that the fluctuations in the hyperfine field arose from the exchange scattering of the conduction electrons by the rare-earth impurity.

The favorable agreement between experiment and theory in the cases of $\mathrm{Er}$ and $\mathrm{Dy}$ in $\mathrm{Ag}$ suggests that it may be possible to use IMPAC techniques to obtain estimates of the rare-earth-conductionelectron interactions in cases where the EPR experiments are not feasible. However for such a program to work, information must be available about the crystalline-field parameters associated with the rare-earth sites. As emphasized in Sec. III any quantitative analysis of $\tau_{c}$ requires the use of crystal field wave functions and energy levels.

\section{APPENDIX}

In this Appendix the calculation of $\tau_{\Gamma}$, the correlation time for the $\Gamma$ manifold, is outlined. As noted $\tau_{\Gamma}$ is equal to $T_{2}$, the EPR linewidth for the $\Gamma$ manifold. Calculations of $1 / T_{2}$ appropriate to the present problem have appeared in the literature. ${ }^{23,24}$ However, these have been limited to very low temperatures where only intramanifold transitions are important. In the temperature range of this experiment, $77 \lessgtr T \$ 450 \mathrm{~K}$, both intra- and inter-manifold transitions must be included since $k T \gtrsim$ typical crystal-field splittings.

Since only the magnitude of $\tau_{\Gamma}$ relative to the magnitude of $T_{2}$ for the ground multiplet is of interest, it is sufficient to take the interaction responsible for the fluctuations in $\vec{J}$ to be of the form

$$
H_{\text {Int }}(t)=\overrightarrow{\mathrm{J}} \cdot \overrightarrow{\mathrm{H}}(t),
$$

where $\overrightarrow{\mathrm{H}}(t)$ is a random operator with the properties

$$
\begin{aligned}
& \langle\overrightarrow{\mathrm{H}}(t)\rangle=0, \\
& \left\langle H_{i}(t) H_{j}\left(t^{\prime}\right)\right\rangle=\delta i j\left\langle H_{i}^{2}\right\rangle f\left(t-t^{\prime}\right) .
\end{aligned}
$$

The normalized correlation function $f(t)$ is characterized by a decay time on the order of $\hbar / E_{F}$, where $E_{F}$ is the Fermi energy of the host. The appearance of the scalar product in (A1) is indicative of the fact that the role of $H_{\text {Int }}$ is to simulate the exchange interaction between the rareearth ion and the conduction electrons.

The analysis begins with the expansion of the operator $\hat{J}_{z}(t)$ :

$$
\hat{J}_{z}(t)=e^{-i H} C F^{t / \hbar} J_{z}(t) e^{i H} C F^{t / \hbar} .
$$

To second order in $H_{\text {Int }}$ this becomes

$$
\begin{aligned}
\hat{J}_{z}(t)= & J_{z}+(i / \hbar) \int_{0}^{t} d t^{\prime}\left[\hat{H}_{\mathrm{Int}}\left(t^{\prime}\right), J_{z}\right] \\
& +(1 / \hbar)^{2} \int_{0}^{t} d t^{\prime} \int_{0}^{t} d t^{\prime \prime} \hat{H}_{\mathrm{Int}}\left(t^{\prime}\right) J_{z} \hat{H}_{\mathrm{Int}}\left(t^{\prime \prime}\right)
\end{aligned}
$$




$$
\begin{aligned}
-(1 / \hbar)^{2} \int_{0}^{t} d t^{\prime} & \int_{0}^{t \prime} d t^{\prime \prime}\left\{\hat{H}_{\mathrm{Int}}\left(t^{\prime}\right) \hat{H}_{\mathrm{Int}}\left(t^{\prime \prime}\right) J_{z}\right. \\
& \left.+J_{\mathrm{z}} \hat{H}_{\mathrm{Int}}\left(t^{\prime \prime}\right) \hat{H}_{\mathrm{Int}}\left(t^{\prime}\right)\right\}, \quad
\end{aligned}
$$

where

$$
\hat{H}_{\text {Int }}(t)=e^{-i H_{C F} t / \hbar} H_{\text {Int }}(t) e^{+i H_{C F} t / \hbar} \text {. }
$$

An expression for $\tau_{\Gamma}$ is obtained from the sum

$$
\begin{aligned}
\sum_{n(\Gamma), n^{\prime}(\Gamma)}\left\langle\left\langle\Gamma n(\Gamma)\left|J_{z}\right| n^{\prime}(\Gamma) \Gamma\right\rangle\right. & \\
& \left.\times\left\langle\Gamma n^{\prime}(\Gamma)\left|\hat{J}_{z}(t)\right| n(\Gamma) \Gamma\right\rangle\right\rangle,
\end{aligned}
$$

$1-x \approx e^{-x}$, leads to the desired result

$$
\begin{aligned}
& \sum_{n(\Gamma), n^{\circ}(\Gamma)}\left\langle\left\langle\Gamma n(\Gamma)\left|J_{z}\right| n^{\prime}(\Gamma) \Gamma\right\rangle\right. \\
& \left.\quad \times\left\langle\Gamma n^{\prime}(\Gamma)\left|\hat{J}_{z}(t)\right| n(\Gamma) \Gamma\right\rangle\right\rangle \\
& =\sum_{n(\Gamma), n^{\prime}(\Gamma)}\left|\left\langle\Gamma n(\Gamma)\left|J_{z}\right| n^{\prime}(\Gamma) \Gamma\right\rangle\right|^{2} e^{-|t| / \tau_{\Gamma},}
\end{aligned}
$$

where

$$
\frac{1}{\tau_{\Gamma}}=\frac{\sum_{n(\Gamma), n^{0}(\Gamma)}\left\langle\Gamma n(\Gamma)\left|J_{z}\right| n^{\prime}(\Gamma) \Gamma\right\rangle D(\Gamma)_{n^{\prime}(\Gamma), n(\Gamma)}}{\sum_{n(\Gamma), n^{0}(\Gamma)}\left|\left\langle\Gamma n(\Gamma)\left|J_{z}\right| n^{\prime}(\Gamma) \Gamma\right\rangle\right|^{2}},
$$

where the second brackets denote an average over the fluctuations in $\overrightarrow{\mathrm{H}}$. The usual approximation, with

$$
\begin{aligned}
& D(\Gamma)_{n^{\circ}(\Gamma), n(\Gamma)}=T C_{e 1}\left\langle\left(\sum _ { \Gamma ^ { \prime } } \sum _ { n ( \Gamma ^ { \prime } ) , n ^ { \prime \prime } ( \Gamma ) } \left[\left\langle\Gamma n^{\prime}(\Gamma)|\hat{h} \cdot \vec{J}| n\left(\Gamma^{\prime}\right) \Gamma^{\prime}\right\rangle\left\langle\Gamma^{\prime} n\left(\Gamma^{\prime}\right)|\hat{h} \cdot \vec{J}| n^{\prime \prime}(\Gamma) \Gamma\right\rangle\right.\right.\right. \\
& \times\left\langle\Gamma n^{\prime \prime}(\Gamma)\left|J_{z}\right| n(\Gamma) \Gamma\right\rangle+\left\langle\Gamma n^{\prime}(\Gamma)\left|J_{z}\right| n^{\prime \prime}(\Gamma) \Gamma\right\rangle\left\langle\Gamma n^{\prime \prime}(\Gamma)|\hat{h} \cdot \vec{J}| n\left(\Gamma^{\prime}\right) \Gamma^{\prime}\right\rangle \\
& \left.\times\left\langle\Gamma^{\prime} n\left(\Gamma^{\prime}\right)|\hat{h} \cdot \vec{J}| n(\Gamma) \Gamma\right\rangle\right] \frac{\left(E_{\Gamma^{\prime}}-E_{\Gamma}\right) / k T}{e^{\left(E_{\Gamma^{\prime}}-E_{\Gamma^{\prime}}\right) / k T}-1}-2 \sum_{\Gamma^{\prime}} \sum_{n\left(\Gamma^{\circ}\right), n^{\prime}\left(\Gamma^{\circ}\right)}\left\langle\Gamma n^{\prime}(\Gamma)|\hat{h} \cdot \vec{J}| n\left(\Gamma^{\prime}\right) \Gamma^{\prime}\right\rangle \\
& \left.\left.\times\left\langle\Gamma^{\prime} n\left(\Gamma^{\prime}\right)\left|J_{z}\right| n^{\prime}\left(\Gamma^{\prime}\right) \Gamma^{\prime}\right\rangle\left\langle\Gamma^{\prime} n^{\prime}\left(\Gamma^{\prime}\right)|\hat{h} \cdot \vec{J}| n(\Gamma) \Gamma\right\rangle \frac{\left(E_{\Gamma^{\prime}}-E_{\Gamma}\right) / k T}{e^{\left(E^{\prime} \Gamma^{\prime-E} \Gamma^{\prime} / k T\right.}-1}\right)\right\rangle_{\hat{h}},
\end{aligned}
$$

where $\hat{h}$ is a unit vector and the symbol $\langle\cdots\rangle_{\hat{h}}$ denotes an average over all directions of $\hat{h}$. The constant $C_{e 1}$ appearing in front of (A9) is temperature independent and is proportional to the integral of $\left\langle H_{i}(t) H_{i}(0)\right\rangle$. The sum on $\Gamma^{\prime}$ is over all crystalfield multiplets. The terms with $\Gamma^{\prime}=\Gamma$ characterize the elastic scattering of the electrons, whereas those with $\Gamma^{\prime} \neq \Gamma$ describe inelastic processes.

One feature of (A9) which merits special comment is the presence of the factors

$$
\frac{\left(E_{\Gamma^{\prime}}-E_{\Gamma}\right) / k T}{e^{\left(E_{\Gamma^{\prime}}{ }^{-E} \Gamma^{j / k T}-1\right.}}
$$

They appear when the fluctuation-dissipation theorem is introduced to convert the integral over the correlation function to an expression involving the imaginary part of the dynamic susceptibility associated with the operator $\overrightarrow{\mathrm{H}}$, or equivalently, the imaginary part of the dynamic spin susceptibility of the electron gas. ${ }^{25,26}$ In obtaining $\tau_{\Gamma}$ in the form shown, explicit use has been made of the fact that the imaginary part of the spin susceptibility is temperature independent for $k T \ll E_{F}$ and a linear function of $\omega$ for $\hbar \omega \ll E_{F} .{ }^{25,26}$ Finally it should be pointed out that $\tau_{c}$ can be written

$$
\tau_{c}=\left[\frac{1}{3} J(J+1)\right]^{-1} \sum_{\Gamma} \tau_{\Gamma} P_{\Gamma} \operatorname{Tr}_{\Gamma}\left(J_{z}\right)_{\Gamma}^{2}
$$

while $\tau_{\Gamma}$ takes the form

$$
\frac{1}{\tau_{\Gamma}}=\frac{\operatorname{Tr}_{\Gamma}\left(J_{z}\right)_{\Gamma} D(\Gamma)}{\operatorname{Tr}_{\Gamma}\left(J_{z}\right)_{\Gamma}^{2}},
$$

where $\operatorname{Tr}_{\Gamma}$ denotes a trace over the states of the $\Gamma$ manifold and $\left(J_{z}\right)_{\Gamma}$ is the projection of the angular-momentum operator onto this manifold. The presence of the traces in (A10) and (A11) indicate that the results are insensitive to noncubic perturbations as long as there is negligible admixing of states from other manifolds.
*Research supported in part by the National Science Foundation, U.S. Atomic Energy Commission, and Conselho Nacional de Pesquisas, Brazil.

${ }^{\dagger}$ Present address: Department of Nuclear Physics, Australian National University, Canberra, A.C.T., Australia.

${ }^{\ddagger}$ On leave from: Instituto de Fisica, Porto Alegre, Brazil.

${ }^{\S}$ Present address: University of Wisconsin, Department of Physics, Superior, Wisc. 54880.

${ }^{1}$ J. C. Waddington, K. A. Hageman, S. Ogaza, D. Kiss, B. Herskind, and B. I. Deutch, in Nuclear Reactions Induced by Heavy Ions, edited by R. Bock and W. R. Hering (North-Holland,
Amsterdam, 1970), p. 438.

${ }^{2}$ P. Ryge, H. W. Kugel, and R. R. Borchers, in Hyperfine Interactions in Excited Nuclei, edited by G. Goldring and R. Kalish (Gordon and Breach, New York, 1971), p. 1043.

${ }^{3}$ B. Herskind, in Ref. 2, p. 987.

${ }^{4}$ A. Abragam and R. V. Pound, Phys. Rev. 92, 943 (1953).

${ }^{5}$ C. Scherer (unpublished).

${ }^{6}$ D. Davidov, R. Orbach, L. J. Tao, and E. P. Chock, Phys. Lett. A 34, 379 (1971).

${ }^{7}$ D. Davidov, R. Orbach, C. Rettori, D. Shaltiel, and L. J. Tao, Phys. Lett. A 35, 339 (1971). 
${ }^{8}$ D. E. Murnick, in Hyperfine Interactions, edited by A. J. Freeman and R. B. Frankel (Academic, New York, 1967), p. 637.

${ }^{9}$ L. Grodzins, in Hyperfine Structure and Nuclear Radiation, edited by E. Matthias and D. A. Shirley (North-Holland, Amsterdam, 1968), p. 607.

${ }^{10}$ G. M. Heestand, R. R. Borchers, B. Herskind, L. Grodzins, R. Kalish, and D. E. Murnick, Nucl. Phys. A. 133, 310 (1969).

${ }^{11}$ J. Lindhard, M. Scharff, and H. E. Schiott, K. Dan. Vidensk. Selsk. Mat.-Fys. Medd. 33 (14), 10 (1963).

${ }^{12}$ B. I. Deutch and G. M. Heestand, in Angular Correlations in Nuclear Disintegrations, edited by $\mathbf{H}$. Van Krugten and $\mathbf{B}$. Van Nooijen (Rotterdam U. P., Groningen, The Netherlands, 1970), p. 487.

${ }^{13}$ P. Ryge, Ph.D. thesis (University of Wisconsin, Madison, 1971) (unpublished).

${ }^{14}$ H. Frauenfelder and R. M. Steffen, in Alpha-, Beta-, and Gamma-Ray Spectroscopy, edited by K. Siegbahn

(North-Holland, Amsterdam, 1968), p. 997.

${ }^{15}$ Nuclear Data, edited by K. Way (Academic, New York, 1965), Vol. 1.

${ }^{16} \mathrm{C}$. Günther, G. Strube, U. Wehmann, W. Engels, H. Blumberg,
H. Luig, R. M. Lieder, E. Bodenstedt, and H. J. Körner, Z. Phys. 183, 472 (1965).

${ }^{17}$ The value of $A\left({ }^{163} \mathrm{Dy}\right)$ was obtained from the EPR data for $\mathrm{Dy}^{3+}$ in Rh [D. Davidov, R. Orbach, C. Rettori, D. Shaltiel,

L. J. Tao, and B. Ricks, Phys. Lett. A 37, 361 (1971)].

${ }^{18}$ L. J. Tao, D. Davidov, R. Orbach, and E. P. Chock, Phys. Rev. B 4, 5 (1971)

${ }^{19}$ Values of $g_{n}$ were obtained from the table of nuclear moments prepared by V. S. Shirley which appears in Ref. 2, p. 1255.

${ }^{20}$ A. R. Chuhran, A. Li-Scholz, and R. L. Rasera, in Ref. 2, p. 464.

${ }^{21}$ G. Williams and L. L. Hirst, Phys. Rev. 185, 407 (1969).

${ }^{22}$ K. R. Lea, M. J. M. Leask, and W. P. Wolf, J. Phys. Chem. Solids 23, 1381 (1962).

${ }^{23}$ C. R. Burr and R. Orbach, Phys. Rev. Lett. 19, 1133 (1967).

${ }^{24}$ R. Orbach and H. J. Spencer, Phys. Lett. A 26, 457 (1968).

${ }^{25}$ T. Moriya, J. Phys. Soc. Jap. 18, 516 (1963).

${ }^{26}$ T. Izuyama, D-J. Kim, and R. Kubo, J. Phys. Soc. Jap. 18, 1025 (1963).

\title{
Nuclear Magnetic Resonance of ${ }^{11} \mathrm{~B}$ at the Three Boron Sites in Rare-Earth Tetraborides
}

\author{
J. H. N. Creyghton, ${ }^{*}$ P. R. Locher, and K. H. J. Buschow \\ Philips Research Laboratories, Eindhoven, The Netherlands
}

(Received 13 October 1972)

\begin{abstract}
An experimental study has been made of the ${ }^{11} \mathrm{~B}$ Knight shift at the three different crystallographic boron sites in polycrystalline $\mathrm{NdB}_{4}$. The three central transitions strongly overlap and cannot be analyzed, but it was possible to derive three different Knight shifts $[+0.33(3),+0.26(4)$, and $+0.23(3)$ $\%$ at $77 \mathrm{~K}$ for the sites $4 e, 8 j$, and $4 h$, respectively, uncorrected for pseudocontact and demagnetization fields] from the satellites, which are separated from each other by their different quadrupole interactions $\left[v_{Q}=420(6) \mathrm{kHz}\right.$ and $\eta=0$ for site $4 e, v_{Q}=443(10) \mathrm{kHz}$ and $\eta=0.51(2)$ for site $8 j$, and $v_{Q}=622(6) \mathrm{kHz}$ and $\eta<0.05$ for site $4 h$, at both 296 and $77 \mathrm{~K}$ ]. A detailed account is given of the method of extracting the various shift and quadrupole parameters from the powder satellite spectra. We first analyzed $\mathrm{LaB}_{4}$, in which the shifts are zero $( \pm 0.02 \%)$ with respect to $\mathrm{Na}_{2} \mathrm{~B}_{2} \mathrm{O}_{4}$ and in which the quadrupole interactions are $\nu_{Q}=343(4) \mathrm{kHz}$ and $\eta=0$ for $4 e, \nu_{Q}=412(4) \mathrm{kHz}$ and $\eta=0.53(1)$ for $8 j$, and $\nu_{Q}=544(4) \mathrm{kHz}$ and $\eta=0.045(20)$ for $4 h(4-300 \mathrm{~K})$. In $\mathrm{NdB}_{4}$, relatively large anisotropic contributions to the shifts are found to originate mainly from dipolar fields due to the rare-earth magnetic moments. After correction for pseudocontact shifts, the isotropic hyperfine fields at boron per unit spin $S$ are about $-2 \mathrm{kG}$. Preliminary measurements on $\mathrm{GdB}_{4}$ and $\mathrm{HoB}_{4}$ give the same sign for this field. An attempt is made to fit the observed isotropic shifts in $\mathbf{N d B}_{4}$ within the Ruderman-Kittel-Kasuya-Yosida scheme.
\end{abstract}

\section{INTRODUCTION}

It is well known that the localized $4 f$ moments in rare-earth intermetallic compounds interact via the conduction electrons. For a description of magnetic properties, the Ruderman-Kittel-KasuyaYosida (RKKY) model $^{1}$ is often used. One of the consequences of this model is a nonuniform conduction-electron-spin polarization which, in the case of crystallographically inequivalent sites, can lead to a difference in spin polarization at these sites.
In principle, Knight-shift measurements of nuclei of nonmagnetic atoms such as ${ }^{27} \mathrm{Al}$ can give experimental evidence of such a nonuniform conductionelectron-spin polarization. The orthorhombic compounds $R_{3} \mathrm{Al}_{11}$ ( $R$ is a rare earth) have been studied ${ }^{2}$ for this purpose but they are complicated as they have two $R$ and four $\mathrm{Al}$ positions, whereas only two overlapping nuclear-magnetic-resonance (NMR) lines were observed.

In some respects the presently investigated tetragonal $R \mathrm{~B}_{4}$ compounds seemed to be better suited for the purpose. Their structure gives rise 